\title{
Inflammatory status affects the antitumor activity of poly-epitope-peptide vaccination against the thymidylate synthase in metastatic colo-rectal cancer patients enrolled in TSPP/VAC-1 Phase Ib trial
}

\author{
Pierpaolo Correale ${ }^{1}$, Valerio Nardone ${ }^{1}$, Cirino Botta ${ }^{2}$, Elodia Martino ${ }^{1 *}$, Pierpaolo Pastina ${ }^{1}$, Cristina Ulivieri ${ }^{3}$, \\ Maria Grazia Rossetti ${ }^{4}$, Antonella Fioravanti ${ }^{5}$, Claudia Gandolfo ${ }^{6}$, Francesco Carbone ${ }^{7}$, Tatiana Cosima Baldari ${ }^{3}$, \\ Pierosandro Tagliaferri ${ }^{2}$, Luigi Pirtoli ${ }^{1}$, Maria Grazia Cusi ${ }^{6}$
}

From 30th Annual Meeting and Associated Programs of the Society for Immunotherapy of Cancer (SITC 2015) National Harbor, MD, USA. 4-8 November 2015

Thymidylate synthase (TS) is a tumor-associated-enzyme crucial for DNA replication and inhibited by 5 '-fluorouracil. TSPP is a previously characterized anticancer polyepitope peptide vaccine to TS (Correale P, JNCI 2005 97:1437). TSPP/VAC-1 is a three-arm dose-finding PhaseIb trial aimed to test in pretreated-advanced cancer patients, TSPP-vaccination alone ( $\operatorname{arm~A})$, together with GM-CSF and low dose Aldesleukine (arm B), or together with chemo-immunotherapy according to the GOLFIG regimen (Correale P, JCO, 2005, 23:8950) (arm C). TSPP resulted safe, its MTD was not achieved, while its mosteffective-biological-dose was $300 \mu \mathrm{g}$. As the most promising antitumor effects of TSPP were observed in colo-rectal cancer (mCRC) patients (Cusi MG, CIIT, 2015, epub), we decided of carrying-out a new study to evaluate in this subset of patients, the potential ability of a predefined panel of markers to predict their antitumor response to TSPP. We thus evaluated $41 \mathrm{mCRC}$ patients, 20 males and 21 females, with a good performance status, enrolled between May 2011 and Jan 2013. Our parameters were correlated with progression free survival (PFS) and overall survival (OS) by performing a Kaplan Meier analysis. The baseline marker values were divided in two groups according to their median values, while the changes relative to baseline values (post-treatment values) were divided according to a fold ratio $\leq$ or $>1$. Patients' PFS and OS were 6.9 and 11.3 months, respectively; there were no significant differences in PFS and OS correlated with treatment arm (A vs. B vs. C), number and type of previous treatments, sex, age, TS expression, HLA2.1 haplotype or expression of peripheral CTLs, regulatory-T cells, centraland effector-memory-T cells. Patients bearing K-ras mutations, showed a trend to a shorter PFS (p:0.051) and no differences in OS $(\mathrm{p}=0.16)$. Patients' outcome was instead, inversely correlated with performance status (ECOG 0-1 vs. 2; PFS, p1, OS, p:0.039). These results suggest that inflammatory status and autoimmunity may affect TSPP antitumor activity in mCRC patients. These results deserve to be considered for the design of new studies.

\begin{abstract}
Authors' details
${ }^{1}$ Unit of Radiotherapy and Unit of Radiology, Department of Oncology, Siena, Italy. ${ }^{2}$ Department of Experimental and Clinical Medicine, "Magna Graecia" University and Medical Oncology Unit, Fondazione Tommaso Campanella", Catanzaro, Italy. ${ }^{3}$ Department of Life Sciences, Siena University, Siena, Italy. ${ }^{4}$ Unit of Pharmacy, Siena University Hospital, Siena, Italy. ${ }^{5}$ Unit of Rheumatology, Department of Clinical Medicine and Immunologic Sciences, Siena, Italy. ${ }^{6}$ Microbiology Unit, Department of Medical Biotechnology, Siena, Italy. ${ }^{7}$ Unit of Radiology, Siena Hospital, Siena, Italy.
\end{abstract}




\begin{tabular}{|c|c|c|c|c|}
\hline Parameter & Endpoint & Number of patients & Mean Value & Significance \\
\hline ECOG & PFS & $\begin{array}{l}\text { ECOGO-1:29 } \\
\text { ECOG 2: } 12\end{array}$ & $\begin{array}{l}\text { ECOG 0-1:9.31+-2.27 } \\
\text { ECOG 2: } 2.41+-0.43\end{array}$ & p 00.001 \\
\hline KRAS & PFS & $\begin{array}{l}\text { Wud Type:22 } \\
\text { Mat: } 19\end{array}$ & $\begin{array}{l}\text { WT: } 8.77+-2.29 \\
\text { Mht: } 4.68+-1.58\end{array}$ & P:0.051 \\
\hline NLR & PFS & $\begin{array}{l}\text { Usder median vahue } 20 \\
\text { Over median value: } 20\end{array}$ & $\begin{array}{l}\text { Under: } 11.1+-3.18 \\
\text { Over: } 3.80+-0.6\end{array}$ & $\mathrm{p}: 0.010$ \\
\hline CRP & PFS & $\begin{array}{l}\text { Usder median valbe } 20 \\
\text { Over median value: } 20\end{array}$ & $\begin{array}{l}\text { Under.11.25+-3.17 } \\
\text { Over } 3.65+-0.54\end{array}$ & $\mathrm{p}: 0.005$ \\
\hline Post-treatment neutrophil coumt changes & PFS & $\begin{array}{l}\text { Ratio } \leq 1: 16 \\
\text { Ratio }>1: 11\end{array}$ & $\begin{array}{l}\text { Ratio } 1: 9.06+-3.12 \\
\text { Ratio }>1: 2.36+-0.59\end{array}$ & $\mathrm{p}: 0.011$ \\
\hline CBA & OS & $\begin{array}{l}\text { Usder median value :19 } \\
\text { Over median value: } 19\end{array}$ & $\begin{array}{l}\text { Under: } 19.10+-4.19 \\
\text { Over: } 7.42+-1.14\end{array}$ & $\mathrm{p}: 0.021$ \\
\hline ECOG & OS & $\begin{array}{l}\text { ECOG } 01: 29 \\
\text { ECOG 2: } 12\end{array}$ & $\begin{array}{l}\text { ECOG 0-1:17.04+-2.9 } \\
\text { ECOG } 2: 3.91+-0.74\end{array}$ & $p 00.001$ \\
\hline NLR & OS & $\begin{array}{l}\text { Usder median vahue } 20 \\
\text { Over median value: } 20\end{array}$ & $\begin{array}{l}\text { Under: } 19.01+-3.97 \\
\text { Over:7.40+/-1.04 }\end{array}$ & $\mathrm{p}: 0.011$ \\
\hline CRP & OS & $\begin{array}{l}\text { Usder median talue } 20 \\
\text { Over median value: } 20\end{array}$ & $\begin{array}{l}\text { Under: } 19.75+-4.1 \\
\text { Over: } 7.40+-1.48\end{array}$ & $\mathrm{p}: 0.002$ \\
\hline ESR & OS & $\begin{array}{l}\text { Usder median value } 20 \\
\text { Over median valve: } 19\end{array}$ & $\begin{array}{l}\text { Under: } 18.92+-3.69 \\
\text { Over: } 7.52+i-1.97\end{array}$ & $\mathrm{p}: 0.002$ \\
\hline LDH & OS & $\begin{array}{l}\text { Usder median vahe } 20 \\
\text { Over median value: } 20\end{array}$ & $\begin{array}{l}\text { Under: } 19.64+-3.88 \\
\text { Over: } 6.60+-0.84\end{array}$ & $\mathrm{p}: 0.001$ \\
\hline ENA & OS & $\begin{array}{l}\text { Usder median value : } 22 \\
\text { Over median value: } 17\end{array}$ & $\begin{array}{l}\text { Under: } 17.30+-3.52 \\
\text { Over: } 8.58+i-2.11\end{array}$ & $\mathrm{p}: 0.023$ \\
\hline III 17 & OS & $\begin{array}{l}\text { Usder median value : } 18 \\
\text { Over median value: } 18\end{array}$ & $\begin{array}{l}\text { Under: } 10.61+-2.82 \\
\text { Over: } 17.83+-3.36\end{array}$ & $\mathrm{p}: 0.074$ \\
\hline IILA & OS & $\begin{array}{l}\text { Usder median talue : } 18 \\
\text { Over median value: } 18\end{array}$ & $\begin{array}{l}\text { Under: } 10.33+-2.8 \\
\text { Over: } 17.49+-3.31\end{array}$ & p:0.049 \\
\hline Post-treatment neutrophil Count changes & OS & $\begin{array}{l}\text { Ratio } \leq 1: 25 \\
\text { Ratio >1: } 14\end{array}$ & $\begin{array}{l}\text { Ratio<1: } 18.51+.3 .38 \\
\text { Ratio>1: } 5.85+-1.29\end{array}$ & p:0.001 \\
\hline Post-treatment $C-A N C A$ changes & OS & $\begin{array}{l}\text { Ratio } \leq 1: 8 \\
\text { Ratio }>1: 13\end{array}$ & $\begin{array}{l}\text { Ratio<1: } 6.12+-1.67 \\
\text { Ratio }>1: 16.76+-4.22\end{array}$ & p:0.039 \\
\hline
\end{tabular}

Figure 1

Published: 4 November 2015

doi:10.1186/2051-1426-3-S2-P443

Cite this article as: Correale et al: Inflammatory status affects the antitumor activity of poly-epitope-peptide vaccination against the thymidylate synthase in metastatic colo-rectal cancer patients enrolled in TSPP/VAC-1 Phase Ib trial. Journal for Immunotherapy of Cancer 2015 3(Suppl 2):P443. 